\title{
PNEUMOCOCCAL CONJUGATE VACCINE AND PNEUMONIA PREVENTION IN CHILDREN WITH CONGENITAL HEART DISEASE
}

\author{
Fortino Solórzano-Santos ${ }^{1}$, Lilia Espinoza-García², Glorinella Aguilar-Martínez ${ }^{3}$, \\ LUISA Beirana-Palencia ${ }^{4}$, Gabriela ECháNIZ-Avilés ${ }^{5}$ AND Guadalupe Miranda-Novales ${ }^{6 *}$
}

${ }^{1}$ Evidence-based Medicine Research Unit, Hospital Infantil de México, "Federico Gómez", Secretaría de Salud, Mexico City; ${ }^{2}$ Department of Pediatrics, Hospital Regional \# 72 Tlalnepantla, Instituto Mexicano del Seguro Social, Tlalnepantla, Mexico City; ${ }^{3}$ Centro Médico de Occidente, Instituto Mexicano del Seguro Social, Guadalajara, Jal.; ${ }^{4}$ Department of Cardiology, Hospital Ángeles, Clínica Londres, Mexico City; ${ }^{5}$ nstituto Nacional de Salud Pública, Cuernavaca, Mor.; ${ }^{6}$ Hospital Epidemiology Research Unit, Health Research Coordination, IMSS, Mexico City. Mexico

\begin{abstract}
Background: A successful strategy to prevent Streptococcus pneumoniae infections is the administration of pneumococcal conjugate vaccines (PCVs). Objective: To analyze the effectiveness of the 7-and 13-valent PCV for the prevention of all-cause pneumonia. Materials and Methods: A retrospective cohort of children younger than 5 years of age, with congenital heart disease (CHD) and different vaccination schedules, was analyzed. History of vaccination was confirmed with verifiable records. The outcome measure was all-cause pneumonia or bronchopneumonia. Protocol was approved by the Institutional Review Board. For comparisons, we used inferential statistics with Chi-square and Fisher's exact test; a $p \leq 0.5$ was considered statistically significant. Relative and absolute risks reduction and number needed to treat were also calculated. Results: A total of 348 patients were included: 196 with two or more doses of PCV (considered the vaccinated group), and 152 in the unvaccinated group. There was a statistically significant difference for pneumonia events $(p<0.001)$ between the vaccinated $(26 / 196)$ and unvaccinated (51/152) groups. The relative risk reduction was $60.5 \%$, and the absolute risk reduction, $20.3 \%$. There were no differences between patients who received two, three or four doses. The number needed to vaccinate to prevent one event of pneumonia was 5 children. Conclusions: At least two doses of PCV in children with CHD reduced the risk of all-cause pneumonia.
\end{abstract}

Key words: Congenital heart disease. Pneumonia. Streptococcus pneumoniae. Vaccine.

\section{INTRODUCTION}

In developing countries, invasive pneumococcal disease remains as one of the main causes of morbidity and mortality in children younger than 5 years, with the highest incidence among children under 18 months of age. Streptococcus pneumoniae is a major bacterial pathogen responsible for a wide spectrum of invasive diseases (pneumonia, bacteremia, and meningitis), as well as non-invasive diseases. In addition to young children, patients with high risk are those with a variety of underlying and chronic conditions (e.g., children with

\section{Corresponding author:}

*Guadalupe Miranda-Novales

Unidad de Investigación en Epidemiología Hospitalaria

Coordinación de Investigación en Salud

Instituto Mexicano del Seguro Social

Av. Cuauhtémoc, 330

Col. Doctores, Del. Cuauhtémoc

C.P 06720, Ciudad de México, México

E-mail: guadalupe.mirandan@imss.gob.mx surgical or functional asplenia, organ transplant, AIDS, primary immunodeficiencies, chronic heart, lung, liver or renal disease, and with cerebrospinal fluid leak $)^{1,2}$.

A successful global strategy to prevent infections by $S$. pneumoniae has been the application of pneumococcal conjugate vaccines (PCVs) ${ }^{3,4}$. In Mexico, the heptavalent PCV was available in 2006 and first administered to a selected high-risk group of children. It was included in 2008 to the National Immunization Program. In private medicine, three doses plus a booster (at 2, 4, 6, and 12 months) were administered,

Received for publication: 03-04-2017

Approved for publication: 30-05-2017

doi: $10.24875 /$ RIC.17002241 
while the National Immunization Program included a primary series of two doses at 2 and 4 months of age and a booster at 12 months of age. The 10-valent PCV (additional serotypes 1,5 , and $7 F$ ) is only available in private medicine, and the 13-valent PCV (additional serotypes 1, 3, 5, 6A, 7F, and 19A) was introduced in 2012 and replaced the 7-valent vaccine in the National Immunization Program in 2013,5.

Studies of the vaccine's effectiveness in healthy children are reported worldwide; some reports include patients with chronic conditions. The objective of this study was to analyze the effectiveness of the 7-valent and 13-valent PCVs for the prevention of pneumonia in a group of children with congenital heart disease (CHD) with different vaccination schedules.

\section{MATERIALS AND METHODS}

A retrospective cohort study was conducted from January 2008 to December 2013, including children younger than 5 years of age with CHD attended at the cardiology department of a tertiary care level hospital in Mexico City. Eligible criteria included history of vaccination with PCVs that could be corroborated by verifiable written records (obligatory vaccination cards and/or hospital patients' database) and available medical charts to obtain the following data: Age, sex, type of CHD, medical visits, hospitalizations, length of hospital stay, and diagnoses of lower respiratory tract infections. The protocol was approved by the Institutional Review Board.

The outcome measure was a diagnosis of pneumonia or bronchopneumonia, established by the patients' treating physicians (cardiologist and pediatrician). Allcause pneumonia (or bronchopneumonia) was chosen as the outcome measure because the confirmation of the etiologic agent by culture is seldom achieved in the pediatric population, and S. pneumoniae is recognized as the most common cause.

A minimum follow-up of 18 months was required for each patient after the first consultation at the hospital. The vaccine dose was counted if it had been administered at least 3 weeks before the onset of the symptoms and signs of lower respiratory tract infection. For the analysis, patients were divided into two main groups according to the number of PCV doses received. Patients who received none or one dose were considered unvaccinated. Exclusion criteria included the absence of verifiable written records of vaccination history and/or an incomplete medical chart.

\section{Statistical analysis}

Descriptive statistics with simple frequencies and percentages were used. For comparisons, we performed inferential statistics using Chi-square and Fisher's exact test. A p $<0.5$ was considered statistically significant. Relative and absolute risk reduction and number needed to treat were also calculated.

\section{RESULTS}

A total of 348 patients were identified during the study period; 196 with two or more doses of the PCV were assigned to the vaccinated group, and 152 to the unvaccinated group ( 22 patients with no dose and 130 patients with one dose). Seven patients died due to complications not related to pneumonia or bronchopneumonia events.

On the first visit to the cardiology department, the median of age was 4 months in the group of 0-1 dose (range 2-13 months) and 2 months (range 2-13 months) in the vaccinated group ( 2 or more doses). The type of $\mathrm{CHD}$ in each group of patients is shown in Table 1.

There were $68 / 152(44.7 \%)$ girls in the unvaccinated group and $107 / 196$ (54.5\%) in the vaccinated group $(p=0.06)$. According to the number of doses received, 130 children received one dose, 119 received two doses, 53 received three doses, and 24 received four doses. The median age at the first dose in those patients who received only one dose was 5 months, and for those who received two or more doses, it was 2 months.

\section{Pneumonia and bronchopneumonia events}

The median age at the first event of pneumonia or bronchopneumonia was 7 months (range 2-14 months) for patients in the unvaccinated group. Among the vaccinated children, the median age was 9.5 months (range 6-26 months) for those who received two doses, 10 months (range 8-12 months) for those who received three doses, and also a median of 10 months (range 9-12 months) for children who received four doses.

In the unvaccinated group, there were 42 cases of pneumonia and 9 cases of bronchopneumonia $(n=51)$, in comparison with 20 cases of pneumonia and 6 cases of bronchopneumonia $(n=26)$ in the vaccinated group: For those who received two doses of vaccine, there were 14 cases of pneumonia and 4 of bronchopneumonia; with three doses, there were 4 cases of pneumonia and 1 of bronchopneumonia; and among children with four doses, there were 2 cases of pneumonia and 1 of bronchopneumonia 
Table 1. Types of CHD in the study population

\begin{tabular}{lccc}
\hline CHD & $\begin{array}{c}\text { Unvaccinated* } \\
\mathbf{n = 1 5 2 ( \% )}\end{array}$ & $\begin{array}{c}\text { Vaccinated }^{+} \\
\mathbf{n = 1 9 6 ( \% )}\end{array}$ & $\begin{array}{c}\text { Total }^{*} \mathbf{1 9}(\%) \\
\mathbf{n}=\mathbf{3 4 8}(\%)\end{array}$ \\
\hline Acyanotic CHD with increased pulmonary blood flow & $95(62.5)$ & $146(74.5)$ & $241(69.2)$ \\
Cyanotic CHD with increased pulmonary blood flow & $45(29.6)$ & $25(12.7)$ & $70(20.1)$ \\
Cyanotic CHD with decreased pulmonary blood flow & $9(5.9)$ & $16(8.2)$ & $25(7.1)$ \\
Acyanotic CHD with normal pulmonary blood flow & $3(2)$ & $9(4.5)$ & $12(3.4)$ \\
\hline
\end{tabular}

*Unvaccinated: $\leq 1$ dose of PCV.

'Vaccinated: $\geq 2$ doses of PCV.

CHD: congenital heart disease; PCV: pneumococcal conjugate vaccine

(Table 2). All patients with pneumonia or bronchopneumonia were hospitalized and received antimicrobial treatment. The etiologic agent was not identified in any of the cases. There was a statistical difference in the lower respiratory tract infections between the vaccinated (26/196) and unvaccinated (51/152) groups $(p<0.001)$. There were no differences in the number of cases of respiratory tract infection between patients who received two, three or four doses.

The risk for pneumonia in patients who received two doses was similar compared with patients who received three or four doses, in all of them $<2$ and there was no statistical significance (Table 3 ).

The relative risk reduction when comparing the unvaccinated with the vaccinated group was $60.5 \%$ (95\% confidence interval [Cl]: 39.7-74), the absolute risk reduction was $20.3 \%$ ( $95 \% \mathrm{Cl}: 11.4-29.2$ ), and the number needed to treat was 5 ( $95 \% \mathrm{Cl}: 3.43-8.77)$.

\section{DISCUSSION}

Despite the reduction on the incidence of invasive pneumococcal diseases following the inclusion of the PCVs to the routine immunization programs ${ }^{7,8}$, patients with comorbidities are still a high-risk group with increased hospitalization events due to invasive pneumococcal disease as well as all-cause pneumonia9. In developing countries, children with congenital heart defects (CHDs) are one of the major risk groups ${ }^{10}$. Frequently the delay in the surgical repair is associated with a higher mortality (around $60 \%$ ) in children admitted to the intensive care unit, with diagnosis of sepsis or pneumonia in $74 \%$ of cases, most of them with a CHD lesion with increased pulmonary blood flow, similar to this cohort ${ }^{11}$.

Despite the recommendation for the vaccination of high-risk groups ${ }^{12}$, the vaccination coverage is suboptimal in many countries. Therefore, several studies have evaluated the impact of different schedules ${ }^{13-17}$, not only in terms of invasive disease but also in immune response, colonization and all-cause pneumonia as an
Table 2. Number of pneumonia or bronchopneumonia events in the vaccinated and unvaccinated groups

\begin{tabular}{|c|c|c|c|c|}
\hline \multirow[t]{3}{*}{ Number of events } & \multicolumn{4}{|c|}{ Number of PCV doses } \\
\hline & \multicolumn{2}{|c|}{$\begin{array}{l}\text { Vaccinated } \\
\text { group }\end{array}$} & \multicolumn{2}{|c|}{$\begin{array}{l}\text { Unvaccinated } \\
\text { group }\end{array}$} \\
\hline & Two & Three & Four & $\leq 1$ \\
\hline Number of patients & 119 & 53 & 24 & 152 \\
\hline $\begin{array}{l}\text { Pneumonia or } \\
\text { bronchopneumonia events* }\end{array}$ & 18 & 5 & 3 & 51 \\
\hline
\end{tabular}

Table 3. Risk of pneumonia according to the number of doses of PCV

\begin{tabular}{lccc}
\hline Number of PCV doses & OR & $95 \% \mathrm{Cl}$ & $\mathrm{p}$ value \\
\hline Two versus three doses & 1.85 & $0.67-5.8$ & 0.33 \\
Two versus four doses & 1.24 & $0.33-4.62$ & 0.51 \\
Two versus three or four doses & 1.62 & $0.67-3.9$ & 0.14 \\
\hline
\end{tabular}

OR: odds ratio; $\mathrm{Cl}$ : confidence interval; $\mathrm{PCV}$ : pneumococcal conjugate vaccine

indirect effect from the vaccine administration. This study was designed to evaluate whether the number of received doses of the PCV had an effect on the number of all-cause pneumonia. Of the total group, $93 \%$ had received one or more doses of the vaccine, but only $22 \%$ had received three or more doses. Invasive infections in infants younger than 60 days of age are uncommon but are still reported with similar frequency before and after the introduction of the PCV in Mexico ${ }^{18}$. In this study, the youngest patient with pneumonia was 2 months old and had not received the first dose. For analysis purposes, the infant was assigned to the unvaccinated group, according to the definition criteria. This case only emphasizes the need to evaluate protection strategies for infants younger than 60 days of age.

These results showed no difference between patients who received two, three or four doses. Rodgers et al. reviewed published data of the immune responses following two infant doses in countries using a $2+1$ schedule. The proportion of responders after two 
PCV-13 doses were $>85 \%$ for most of the 7 common and 6 additional serotypes, except for 6B, 23F, 3, and $6 \mathrm{~A}$, but all serotypes in $>95 \%$ of the participants showed functional activity. Higher immunogenicity was observed in the Mexico study, compared with the European studies ${ }^{17}$. This could explain the lack of differences for children receiving two or more doses.

Possible reasons for incomplete vaccine schedules in this study were the myths around immunization of patients with underlying conditions, mainly in primary care services, especially with cyanotic patients. In tertiary care hospitals, specialists are focused on the CHD and associated comorbidities and underestimate the importance of a complete vaccination status. Children who received only one dose were older than children who completed the schedule (5 vs. 2 months), probably because parents considered that the CHD was the most important problem and did not receive the proper recommendation for opportune immunization, or else decided to postpone the administration of the vaccines.

The study has several limitations. The outcome measure (all-cause pneumonia) is an indirect effect of vaccination, and even though $S$. pneumoniae is the main pathogen in bacterial pneumonia in children younger than 5 years, none of the patients in the study had microbiological confirmation of the lower respiratory tract infection. These recommendations by the US Advisory Committee on Immunization Practices include the administration of four doses of vaccine in the first 18 months ${ }^{12}$. The National Immunization Program in Mexico includes a primary series of two doses at 2 and 4 months of age and a booster at 12 months of age. Therefore, the number of patients who received four doses was small, and the comparisons to other groups do not represent definitive results.

In accord with the information of diverse studies, at least two doses were needed to reduce the probability of a lower pulmonary tract infection.

Considering the clinical difficulties to differentiate heart failure from pneumonia, this diagnostic uncertainty may be reduced if a child receives a complete vaccination schedule. In patients with $\mathrm{CHD}$, reinforcement of the recommendation of PCV vaccination could decrease the number of cases of pneumococcal pneumonia, an important cause of morbidity and mortality.

\section{REFERENCES}

1. Yildirim I, Shea KM, Little BA, Silverio AL, Pelton SI; Members of the Massachusetts Department of Public Health. Vaccination, underlying comorbidities, and risk of invasive pneumococcal disease. Pediatrics. 2015;135:495-503.

2. Pelton SI, Weycker D, Farkouh RA, Strutton DR, Shea KM, Edelsberg J. Risk of pneumococcal disease in children with chronic medical conditions in the era of pneumococcal conjugate vaccine. Clin infect Dis. 2014;59:615-23.

3. Pneumococcal vaccines WHO position paper--2012. Wkly Epidemiol Rec. 2012;87:129-44.

4. Bonner K, Welch E, Elder K, Cohn J. Impact of pneumococcal conjugate vaccine administration in pediatric older age groups in low and middle income countries: A systematic review. PLoS One. 2015;10:e0135270.

5. Echaniz-Aviles G, Soto-Nogueron A, Miranda-Novales G, Carnalla-Barajas MN, Velazquez-Meza ME, Solórzano-Santos F. SIREVA network-mexico. Streptococcus pneumoniae serotypes identified in Mexican children with invasive disease before and after the introduction of PCV7 1993-2012. Arch Med Res. 2015; 46:149-53.

6. Esquema de Vacunación. México: Secretaría de Salud. Available from: Last access on 2017 April 10

7. Ladhani SN, Slack MP, Andrews NJ, Waight PA, Borrow R, Miller E. Invasive pneumococcal disease after routine pneumococcal conjugate vaccination in children, England and Wales. Emerg Infect Dis. 2013;19:61-8.

8. Whitney CG, Pilishvili T, Farley MM, Schaffner W, Craig AS, Lynfield R, et al. Effectiveness of seven-valent pneumococcal conjugate vaccine against invasive pneumococcal disease: A matched case-control study. Lancet. 2006;368:1495-502.

9. Weycker D, Farkouh RA, Strutton DR, Edelsberg J, Shea KM, Pelton SI. Rates and cost of invasive pneumococcal disease and pneumonia in patients with underlying medical conditions. BMC Health Serv Res. 2016;16:182.

10. Fallahzadeh MA, Abdehou ST, Hassanzadeh J, Fallhzadeh F, Fallahzadeh $\mathrm{MH}$, Malekmakan L. Pattern of in-hospital pediatric mortality over a 3-year period at university teaching hospitals in Iran. Indian J Crit Care Med. 2015;19:311-5.

11. Mohsin SS, Haque A, Shaikh AS, Bano S, Hasan BS. Outcome of infants with unrepaired heart disease admitted to the pediatric intensive care unit: Single-center developing country perspective. Congenit Heart Dis. 2014;9:116-21.

12. Robinson $\mathrm{CL}$; Advisory Committee on Immunization Practices (ACIP), ACIP Child/Adolescent Immunization Work Group. Advisory committee on immunization practices recommended immunization schedules for persons aged 0 through 18 years - United States, 2016. MMWR Morb Mortal Wkly Rep. 2016;65:86-7.

13. Loo JD, Conklin L, Fleming-Dutra KE, Knoll MD, Park DE, Kirk J, et al. Systematic review of the indirect effect of pneumococcal conjugate vaccine dosing schedules on pneumococcal disease and colonization. Pediatr Infect Dis J. 2014;33:S161-71.

14. Rückinger S, Dagan R, Albers L, Schönberger K, von Kries R. Immunogenicity of pneumococcal conjugate vaccines in infants after two or three primary vaccinations: A systematic review and meta-analysis. Vaccine. 2011;29:9600-6.

15. Scott P, Rutjes AW, Bermetz L, Robert N, Scott S, Lourenco T, et al. Comparing pneumococcal conjugate vaccine schedules based on 3 and 2 primary doses: Systematic review and meta-analysis. Vaccine. 2011;29:9711-21.

16. Pelton SI, Weycker D, Klein JO, Strutton D, Ciuryla V, Oster G. 7-Valent pneumococcal conjugate vaccine and lower respiratory tract infections: Effectiveness of a 2-dose versus 3-dose primary series. Vaccine. 2010;28:1575-82.

17. Rodgers GL, Esposito S, Principi N, Gutierrez-Brito M, DiezDomingo J, Pollard AJ, et al. Immune response to 13-valent pneumococcal conjugate vaccine with a reduced dosing schedule. Vaccine. 2013;31:4765-74

18. Soto-Noguerón A, Carnalla-Barajas MN, Solórzano-Santos $F$ Arrendondo-García JL, Arzate-Barbosa P, Tinoco-Favila JC, et al. Streptococcus pneumoniae as cause of infection in infants less than 60 days of age: Serotypes and antimicrobial susceptibility. Int J Infect Dis. 2016;42:69-73. 